\title{
NEW ASPECTS IN THE FIELD OF SHIP PROPULSION SHAFT TECHNOLOGY
}

\author{
Dan Drăgan \\ "Dunarea de Jos" University of Galati, \\ Faculty of Naval Architecture, Galati, Domneasca \\ Street, No. 47, 800008, Romania, \\ E-mail: granmar_ro@yahoo.com
}

\author{
Oana-Mirela Jarcă \\ "Dunarea de Jos" University of Galati, \\ Faculty of Naval Architecture, Galati, Domneasca \\ Street, No. 47, 800008, Romania, \\ E-mail: oana.dobrot@ugal.ro
}

\begin{abstract}
This paper will describe new concepts in the development of shipbuilding. The use of these methods in the naval field, delivers performance. Combining these modern and theoretical methods leads to an increase in the reliability of the axis line assembly, an economic efficiency as well as shorter mounting or repairing times.
\end{abstract}

Keywords: Shaft line, stern tube, tests models

\section{INTRODUCTION}

The development of shipbuilding technology is based on the emergence of new methods, alongside the classical ones applied in shipyards. This development was possible due to the emergence of new concepts, materials with new properties, advanced equipment.

Nowadays the application of modern methods is applied on a large scale, leading to high performance. The aspects related to the design, construction, assembly and verification of the axial lines constitute a unitary whole.

The combination of theoretical and practical methods applied to the axial lines has the effect of increasing the reliability of the axis line assembly, economic efficiency, shortening of the assembly and repair time.

In addition, the technological leap has led to new concepts and new rules of classification societies that apply to the naval axis line.

In order to analyze the steps currently being taken in the field of naval axis construction, we will refer to the modern and topical steps, applied in recent years and compare them to the classic technology.

\section{CONSTRUCTION ASPECTS}

The evolution of new technological solutions is materialized both in the axis line as well as in specific local areas.

- Seal of the shaft

Classic sealing systems use oil and "lip seals" aft and fore.

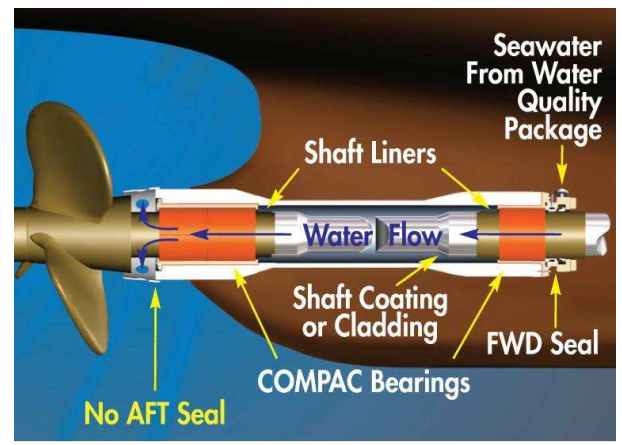

Fig.1. Axis line diagram

Water-tight systems have emerged as an ecological solution and at the same time lead to the replacement of stern sealing. 
Three systems, two (closed and open for the stern tube and one for the knights if necessary) are used.

- Bushing system for water lubrifiction system

The sealing flanges are attached to the stern tube. They are made of bronze and contain the axis bearing.

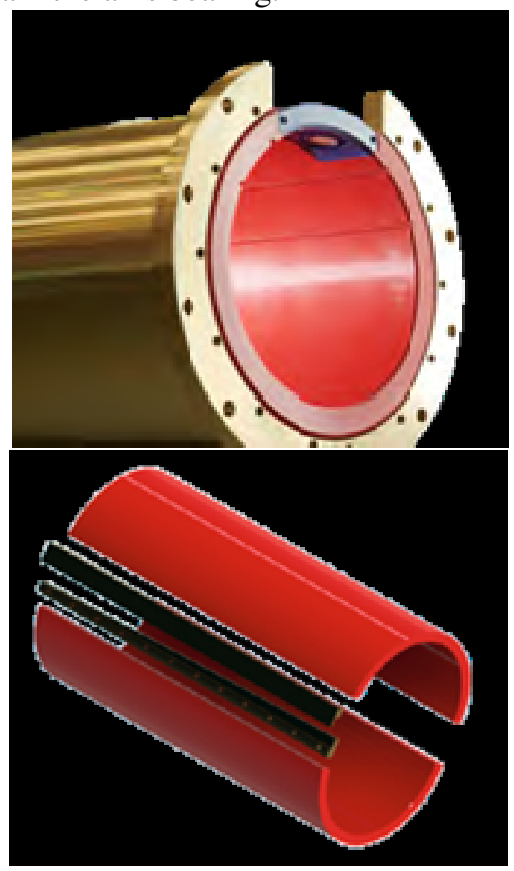

Fig.2. Bushing models

Pieces can be single piece (non split) and two pieces (figure above). In this case it is possible to change them without removing the axis.

-Axis protection.

Under classical conditions, the axis is protected with oil. At the moment there are the following procedures: by plating (metallic coating), synthetic layers, glass fiber, elastomeric rubber.

- UNNET is the aft gasket of aft simering in case of aft sealing.

In order to increase the efficiency of sealing, an additional simering type seal is added. flange.

After dragging on the axis, fix it with a

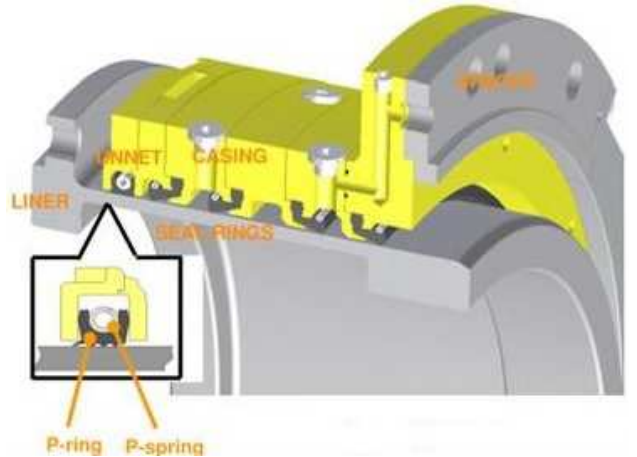

Fig.3. UNNET- additional gasket

New materials

The case where the two bushings are connected by a tube made of elastomeric material is a variant of replacing the classic stern tube with another material. A seal is used in the stern to make a seal with a fixed and movable ring.

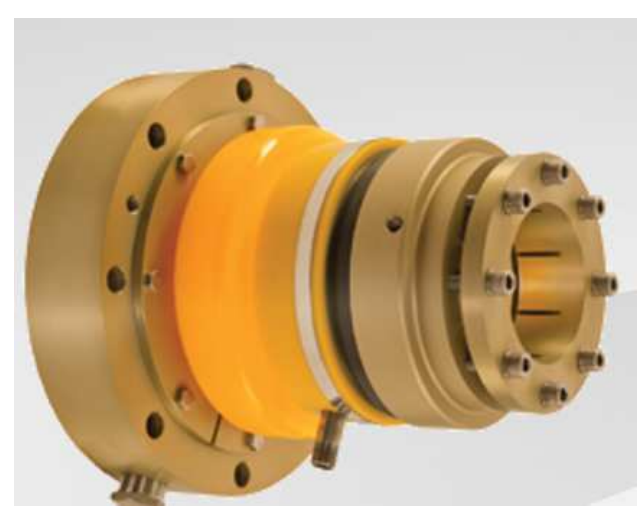

Fig.4. Seal type: face seal

\section{- Assembly / Alignment}

Bushing processing. In most cases, the aft and bow bearings are stored in the tube and mechanically processed. The material used is composite.

In some cases, the bushing is machined to the end, and will be embedded and centered in the tube with the Epocast (Chockfast) material.

The operation is done both at the axis exit of the body and at the knobs to support the axis. By casting the bearing is fixed to the establish quota. 


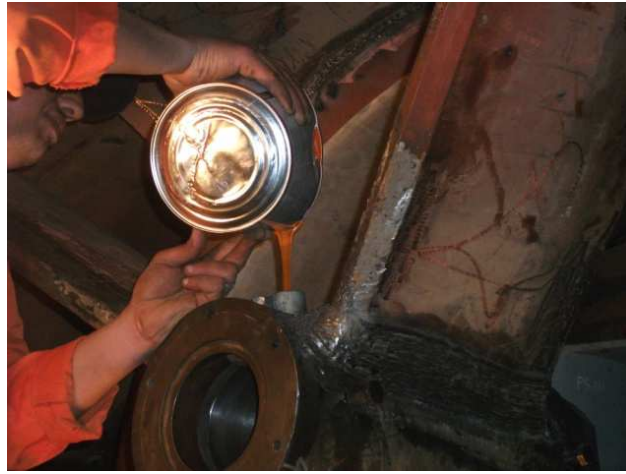

Fig.5. Centering bush by Epocast

\section{- Elevantion}

Piano wire metal elevantion is replaced with laser elevation.

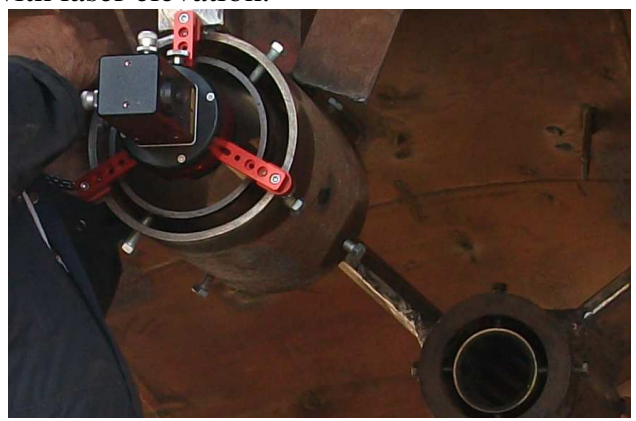

Fig.6. Laser

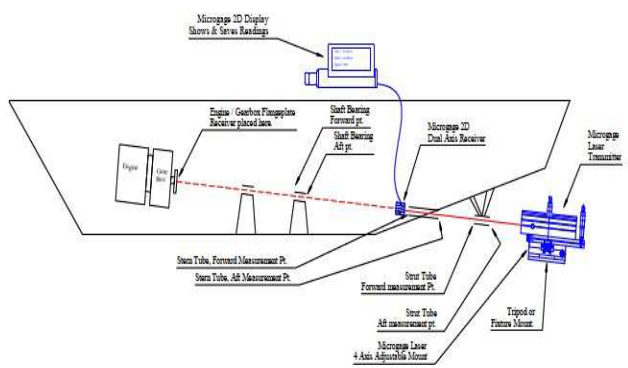

Fig.7. Schematic of the ship's axis line

Of note is the existence of off-set readings through the $2 \mathrm{D}$ remote device.

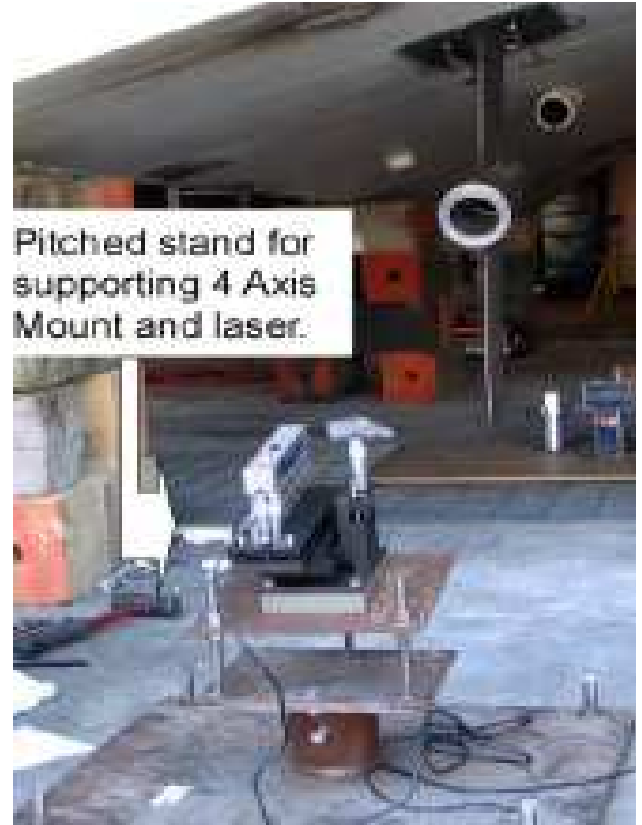

Fig.8. Pitched stand

- Epocast lains

Epocast casting laines are replacing metallic spacers, in order to center the motor, the gearbox. The technology is widely applied in the naval field, based on synthetic resins.

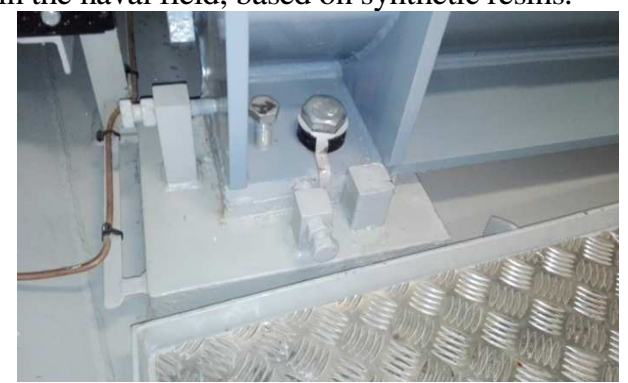

Fig.9. Epocast laine

- Hydraulic coupling - flange coupling replaced by hydraulic.

The cylindrical shaft can be connected to the motor or gear reducer directly or to an intermediate shaft through a hydraulic coupling flange. To mount the coupling, the high pressure oil is injected at more than 2,000 bar between the sleeve and the flange by means of the injectors in order to expand the flange ring. 


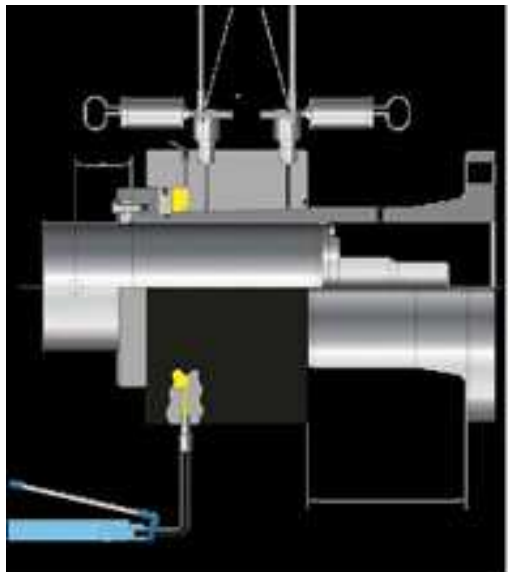

Fig.10. Hydraulic coupling

- Measurement of axial and radial dewaxing, Sag / gap is performed by direct measurements with the micrometers reading on the two flanges. Using the modern method, measuring devices transmit the WiFi signal, allowing for shorter measurements and greater alignment accuracy.

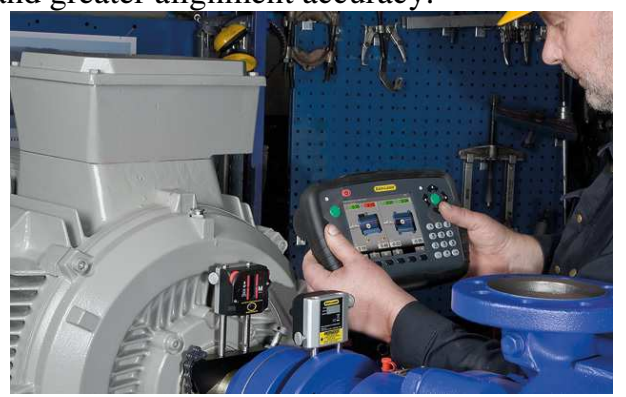

Fig.11. Micrometers reading

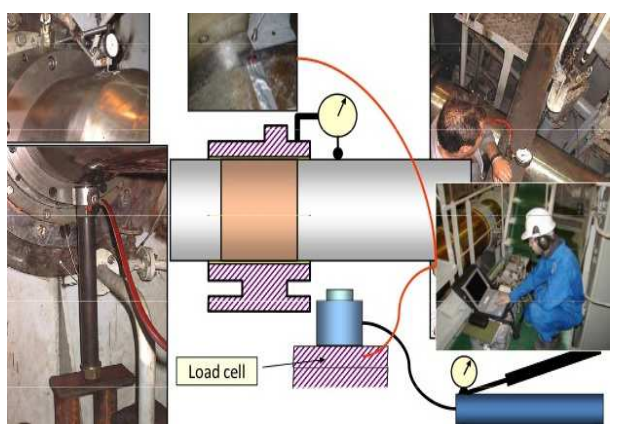

Fig.12. Jack-up test

The second category of check-up is the Jack-up test that highlights the loading of the bearings. For this purpose, data transmission is done online, data processing is based on specialized software.

\section{Check-up}

-Shrinking contact

The shrinking contact, shaft line - propeller hub, is made by pressing and tracking the Prussia blue paint contact $(85 \%)$ at present the trend is $70 \%$ blue, with the mandatory presence of a continuous blue contact ring the extremity of the larger diameter area.

- NDT, Magnetic particles testing

Specifically for determining defects at the tree line is the method of magnetic particles (in liquid suspension), which reveals the defects in the material.

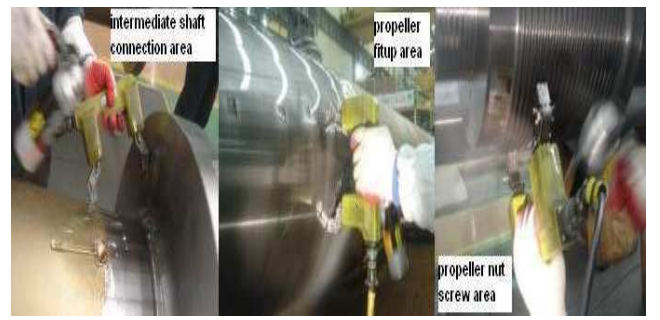

Fig. 13. Magnetic particles method

-Shaft fall

The fall of the propeler spindel shaft, through the wear of bushing support of the spindel is made classically in the upper and lower shaft with the help of the subler at the departure of the ship before the rope guard, the next check being at docking. A permanent device is now being installed to measure the fall of the portal shaft.

Classic - It's observed on secured locking bolts at measiring points with the designated subleth, stored onboard.
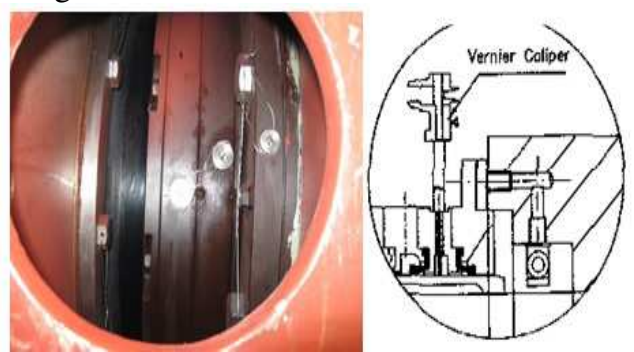

Fig. 14. Measurement method with the scaliper 
At this time, it is possible to measure the "axis fall" on-line during the ship's operation. The presented version contains the device provided with a sensor, mounted on the bushing that transmits the information to a receiver.

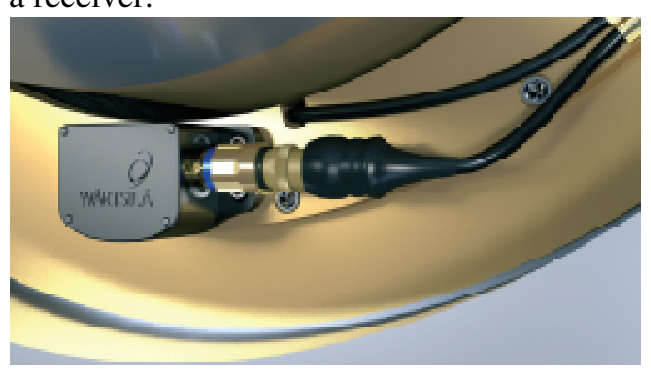

Fig. 15. Sensor frazzle bushing axis propeller

\section{SPECIAL CONSTRUCTIONS}

- Azimuth Propulsion Alignment.

It is a special case that starts with the alignment of the propeller assembly with the body, following the electric motor trim. In fact, it's about aligning two transmission shafts.

Carrying out the propulsion is done in relation to the constructive parts of the ship.

The coupling between the electric motor and the propeller is made with a short line that leads to the rotation of the propeller by an azimuthal mechanism. The rotation of the nozzle and the propeller is done hydraulically.

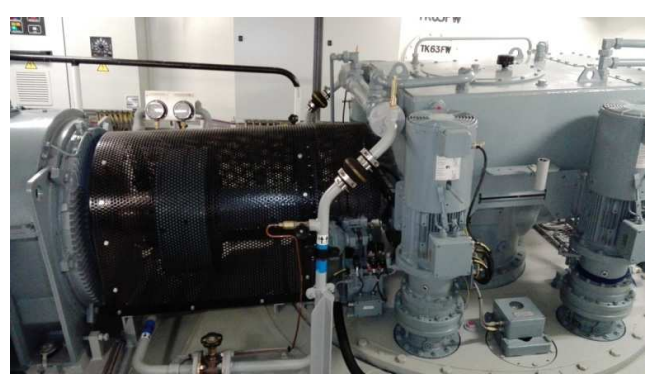

Fig. 16. Shaft line electric engine $-\mathrm{Z}$ transmission

It should be mentioned that in this type of transmission the seal is made of the "lip seal" type, but with a vacuum device which maintains the contact of the rings on the shaft. The centering is initially done with a laser spot fixed to the thruster flange and projected onto the engine flange.

- Watertight sealing systems

Wall-sealing systems allow sealing only in case of damage, flooding of the compartment, when the shaft passes through the walls. The effect is due to the deformation of the hydrogel zone in the presence of water that pushes the lip seal on the shaft. The seal is made of elastomeric material.
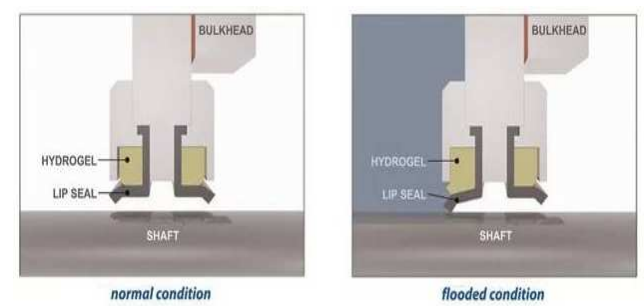

Fig. 17. Watertight sealing systems

\section{RULES}

- DNV Rules: introducing Class 1 and 2 alignment rules.

Alignment of the axial line is a basic option for propulsion systems installed on ships with conventional quarry shapes and facilitates increased performance of the aft bearings during normal operation and operating conditions. The following key requirements for class notation apply:

Aft bearing with several sloaps is mandatory.

Bending moment increase of the propeller ranging from -30 to $+30 \%$ MCR torque in the aft load criteria

The latest measurement techniques for installations observation (laser or equivalent)

Means of warning for immersion of the propeller.

The alignment of the axial line (2) is intended for propulsion systems requiring additional calculations to estimate the hydrodynamic loads of the propeller under the conditions of rotation. Specific 
propulsion systems are found in ships with atypical forms such as asymmetrical aft and pillars.

The following key requirements for class notation (2) apply:

The propulsion-specific hydrodynamic propulsion charge and the transient forces (using CFD)

FE analysis for variable pressure and bearing contact surface

Ship deflections, if any

The alignment conditions of the shaft (1) apply as basic criteria.

-Solas : Safe return to port .

This rule refers to the SOLAS regulation adopted by the IMO, Resolution MSC .216 (82), applicable from 1 July 2010.

The regulation provides for passenger ships of 120 meters or more in length or with three or more major decks to be designed for better survival. This means that in the event of a flood or fire emergency, passengers and crew can stay on board as the ship enters the port in its own capacity. It defines a scenario in which the ship must be able to return to port without evacuating passengers.

\section{CONCLUDING REMARKS}

The prediction of the sailing vessel resistance performance with a good level of accuracy for the initial design stage is an important hydrodynamic problem.

The technological advances related to the shipbuilding line construction have particularities for each type of ship. The chosen solution focuses on safety, maintenance, economic and environmental protection

The implementation of modern solutions is done by the designer, based on the existence of new rules imposed by IMO, Classification Societies,

To be noted that new methods have retained features and concepts of classical methods.

In the above mentioned, some aspects of the naval axis are referred to.

These benchmarks can each be the starting point for new solutions. At the same time, by analyzing the technological evolution, one can establish a trend in the field of application of constructive solutions.

\section{REFERENCES}

[1]. https://cdn.wartsila.com/docs/default-source /product-files/seals-bearings/brochure-o-sbseals-bearings-general.pdfsfvrsn $=3887$ ec 454

[2]. http://www.pne.dk/wp-content/uploads/ 2015/ 02/COMPAC.pdf

[3]. https://rmpropulsion.com/tube-seals/sterntube-seals-spares/compatible-with

[4]. http://www.duwelgroup.com/sites/306/conte nt/docs/Thordon/COMPAC-SeawaterLubricated-Prop-Shaft-Brochure.pdf

[5]. https://www.dnvgl.com/news/dnv-gl-rulesfor-classification-and-offshore-standardsjanuary-2018-edition-107755

[6]. http://www.imo.org/en/KnowledgeCentre/In dexofIMOResolutions/Maritime-SafetyCommittee-(MSC)/Documents/ MSC.216(82).pdf

[7]. http://www.skf.com/binary/83-453272/ SKF18016_Broschuere_BluRun_RZ_18080 10_web.pdf

Paper received on December $20^{\text {th }}, 2018$ 